\title{
Imaging Review of Snowboard Injuries
}

\author{
Cedric Bohyn, MD ${ }^{1}$ Dyan V. Flores, MD ${ }^{1,2}$ Timothy Murray, MD ${ }^{1} \quad$ Bruce Mohr, MD ${ }^{3}$ \\ Mark Cresswell, MD ${ }^{1}$
}

1 Department of Radiology, St Paul's Hospital, University of British Columbia, Vancouver, British Columbia, Canada

2 Institute of Radiology, St. Luke's Medical Center Global City, Metro Manila, Philippines

${ }^{3}$ Whistler Health Care Center, Whistler, British Columbia, Canada

\author{
Address for correspondence Cedric Bohyn, MD, Department of \\ Radiology, AZ Monica Hospital, Antwerp, Belgium \\ (e-mail: cedric_bohyn@hotmail.com).
}

Semin Musculoskelet Radiol 2022;26:54-68.

\begin{abstract}
Keywords

- snowboard

- sports injuries

- imaging

- injury mechanism
\end{abstract}

Snowboarding is popular both as a recreational and competitive winter sport. The origin of snowboarding can be traced back to the United States during the 1960s when Sherman Poppen invented the Snurfer (a word combination of "snow" and "surfer") as a toy for his daughters. Since its inception, snowboarding has experienced continuously increasing popularity. ${ }^{1}$ Snowboarding was first recognized as an official Olympic sport event during the 1998 Winter Olympic Games in Nagano, Japan. Skiing and snowboarding remain the two most popular winter sports to this day. Multiple styles of snowboarding have developed over time, each with its own technique and often specific equipment. Currently, the most common styles both recreational and professional are free ride, freestyle, and alpine racing.

Between 2000 and 2020, the worldwide yearly visiting number of skiers, snowboarders, and other downhill sliding activities remained stable at $\sim 400$ million, as major mature markets experienced reduced growth while other markets are still in the emerging phase. The Alps are the biggest ski destination in the world, capturing $43 \%$ of skier visits. The second biggest destination is America (predominantly North America), accounting for $21 \%$ of skier visits worldwide. $^{2}$
The injury rate of snowboarding is higher than skiing, up to threefold, which is at least partially attributed to the rise of terrain parks. ${ }^{3}$ From 1989 to 1999, the incidence of snowboarding injuries as a component of all injuries related to snow sports increased from $4 \%$ to $56 \%{ }^{3}$

The most common site of snowboarding injury among adults is by far the wrist, accounting for 15 to $28 \%$ of all injuries. ${ }^{4,5}$ The remainder of the top-10 injuries is shoulder soft tissue injury (12\%), ankle sprain (6\%), leg contusion (5\%), medial collateral ligament (MCL) of the knee (4\%), clavicle fracture (4\%), upper body laceration (4\%), concussion (3.5\%), ankle fracture (3\%), and anterior cruciate ligament (ACL) of the knee (2.5\%). ${ }^{5}$ The top-10 snowboard injuries for children and adolescent are wrist injuries (38\%), leg contusion (6\%), concussion (5\%), clavicle fracture (5\%), shoulder soft tissue (3\%), knee MCL (3\%), ankle sprain (3\%), upper body laceration $(3 \%)$, and tibia and ankle fractures $(1-1.5 \%)^{5}$

Children and beginner riders are significantly more likely to get injured. ${ }^{6,7}$ Additional risk factors are unfavorable weather conditions with poor visibility and bad snow conditions (e.g., icy snow or poorly groomed slopes). ${ }^{1,8}$

The injury mechanisms of snowboarding injuries are diverse, but opposite-edging is one of the most frequently
Issue Theme Winter Sports; Guest Editors, Hugue A. Ouellette, BSc, MD, FRCPC and Peter L. Munk, MDCM, FRCPC, FSIR, FFRRCSI(Hon) (c) 2022. Thieme. All rights reserved. Thieme Medical Publishers, Inc., 333 Seventh Avenue, 18th Floor, New York, NY 10001, USA
DOI https://doi.org/ 10.1055/s-0041-1731702. ISSN $1089-7860$. 


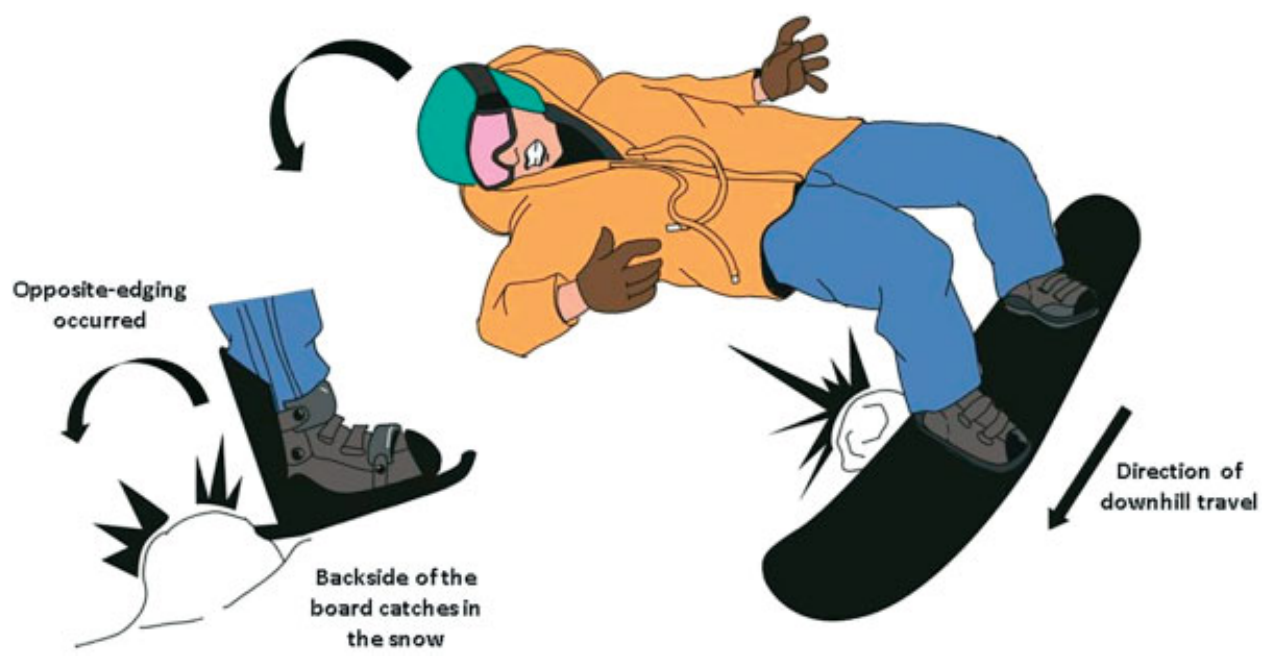

Fig. 1 Graphic illustration of the opposite-edging phenomenon that can result in a fall. This case demonstrates opposite-edging on the backside edge of the board; however, the same can happen on the front-side edge. This injury mechanism typically occurs during downhill runs when the edge of the snowboard opposite to the edge supported on the snow surface (and thus facing the mountain) suddenly and unintentionally catches on the snow surface, resulting in a backward fall (or forward fall if opposite-edging on the front side) with the head and/or upper limbs hitting the snow first.

occurring causes specific to snowboarding and most associated with upper limb injuries (-Fig. 1). ${ }^{1}$

In this review, we provide an overview of common snowboard-related musculoskeletal (MSK) injuries according to anatomical location. The possible underlying mechanisms and radiologic imaging of the different injuries are clarified.

\section{Snowboard Gear, Stance, and Specific Terminology}

The snowboarder typically wears semirigid boots extending to the midcalf that are fastened to moderate tightness. The snowboarder's booted feet are then fastened with rigid bindings (one per foot) to the snowboard. Most binding systems are nonreleasable (contrary to ski bindings), significantly reducing rotational forces to the knees and thus preventing many ACL injuries. ${ }^{9}$

The snowboarder may choose to ride with his bindings arranged so his left foot is in front (regular stance) or his right foot is in front (goofy stance) as per individual preference. A regular stance is generally more common than a goofy stance. More advanced snowboarders "switch" and alternate their leading leg while snowboarding.

The long edge of the board anterior to the snowboarder is known as the front side (or toe side), and the long edge of the board posterior to the snowboarder is known as the backside (or heel side) (-Fig. 2).

The short curved ends of the board are known as the tip (or nose) and the tail, depending on either a regular or goofy stance. The snowboard shape can vary, although the nose and tail are often rounded identically (-Fig. 2 ).

When riding, the snowboard can be oriented in three planes (pitch, roll, and yaw) (-Fig. 2). Yaw is determined by the rotation of the snowboarder around their center of gravity, directing the snowboard to the left or right. Roll is determined by the snowboarder shifting their center of
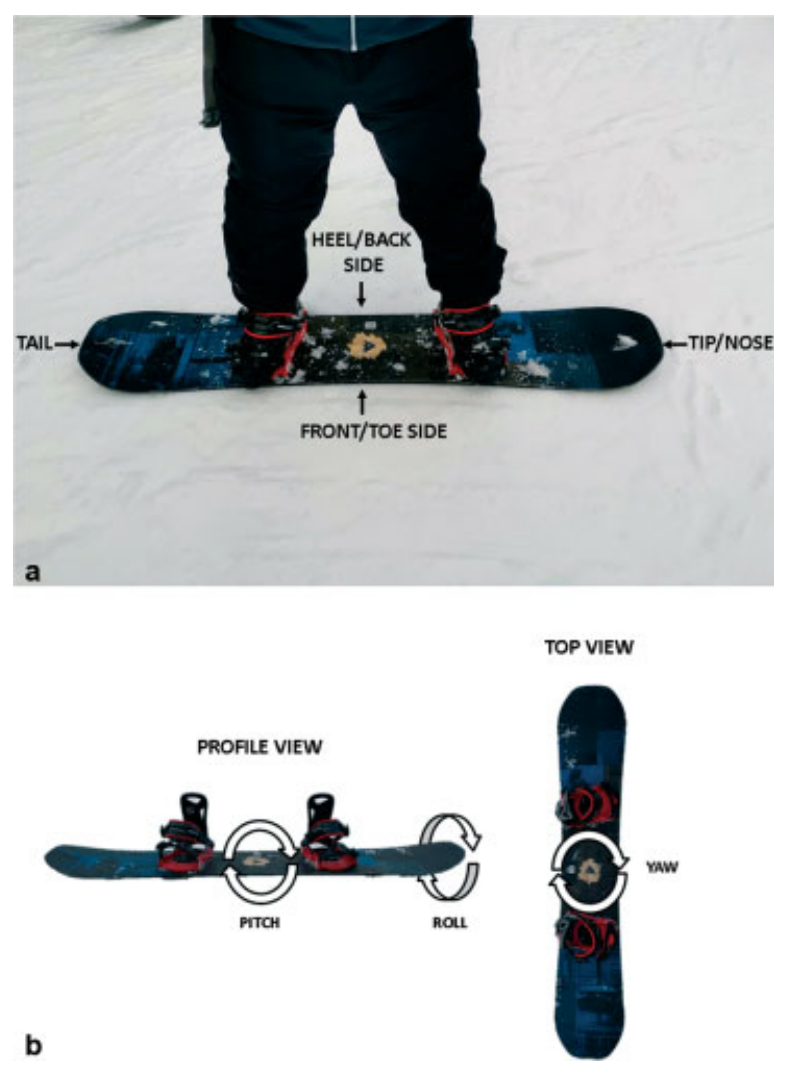

Fig. 2 Snowboard-related terminology. (a) Photograph of snowboarder with a regular stance (left foot first); consequently, the snowboard's short edge is at the side of the left foot is the "tip or nose." (b) The profile view demonstrating the possible rotational axis of the board along the horizontal plane. "Pitch" moves either the nose and tail up or down, whereas "roll" rotates the board around the horizontal plane toward either the toe side or heel side. "Yaw" means rotation around the center of the board with the nose rotating either left or right as shown in the top view. 


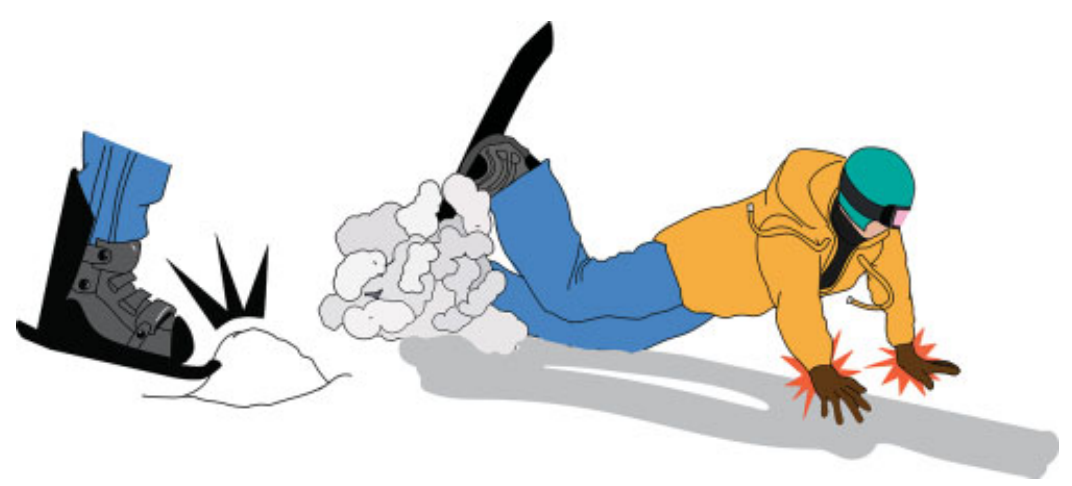

Fig. 3 Illustration of a snowboarder tumbling forward as result of opposite-edging with the toe side catching the snow surface (left). A snowboarder instinctively catches the fall with both arms outstretched, which typically results in upper limb injuries (right).

gravity front side or backside and dictates speed and balance. By increasing the angle of the snowboard with the slope, increased resistance is generated by the scraping effect of the edge of the snowboard on the snow, which acts as a brake. Pitch moves the center of gravity to the front leg (and board tip) or to the back leg (and board tail), which is most relevant in aerial maneuvers (such as flips) and ensures a board-first landing on the snow.

\section{Upper Limb Injuries}

Upper extremity injuries in snowboarders are substantially more frequent compared with skiers. In particular, upper extremity fractures are three times more common compared with skiing. ${ }^{1}$ A likely explanation is that a snowboarder usually falls in an anteroposterior direction with the feet fixed to the board. Because snowboarders do not use ski poles, the outstretched arms and upper body are instinctively used to absorb the (forward or backward) fall ( - Fig. 3).

\section{Distal Radius and Wrist}

The wrist is by far the most commonly injured body part of a snowboarder. ${ }^{5}$ Quinlan et al reported that wrist fractures occurred at 18 times the incidence of skiers. ${ }^{4}$ These wrist fractures are mostly sustained by young and inexperienced snowboarders (-Fig. 4). ${ }^{4}$

A study of 7,430 snowboard injuries (1988-98; Vail, Colorado) found that wrist injuries accounted for $44 \%$ of all upper extremity injuries and $22 \%$ of all snowboard-related injuries. Fractures made up $78 \%$ of wrist injuries. ${ }^{6}$ The Sasaki et al study (1990-97) showed a similar wrist injury rate of $36.4 \%$ of all upper limb injuries, compared with only $9 \%$ of ski injuries. $^{7}$

Distal radius fractures in the setting of snowboarding are not only much more common ( $10 \%$ versus $1 \%$ ), but they also tend to occur in younger individuals compared with skiers. ${ }^{8}$ Sasaki et al reported the mean age of a snowboarder sustaining a distal radius fracture as 21.7 years, compared with 30.8 years for a skier. ${ }^{7}$ These numbers are consistent with the Matsumoto et al study, showing that upper limb injury occurred at an average age of 23 years of age for snowboarders and 27 years for skiers. ${ }^{1}$ According to Quinlan et al, skiers experience proportionally more comminuted and intra-articular wrist fractures, whereas snowboarders sustain rather simple extra-articular fractures. ${ }^{4}$
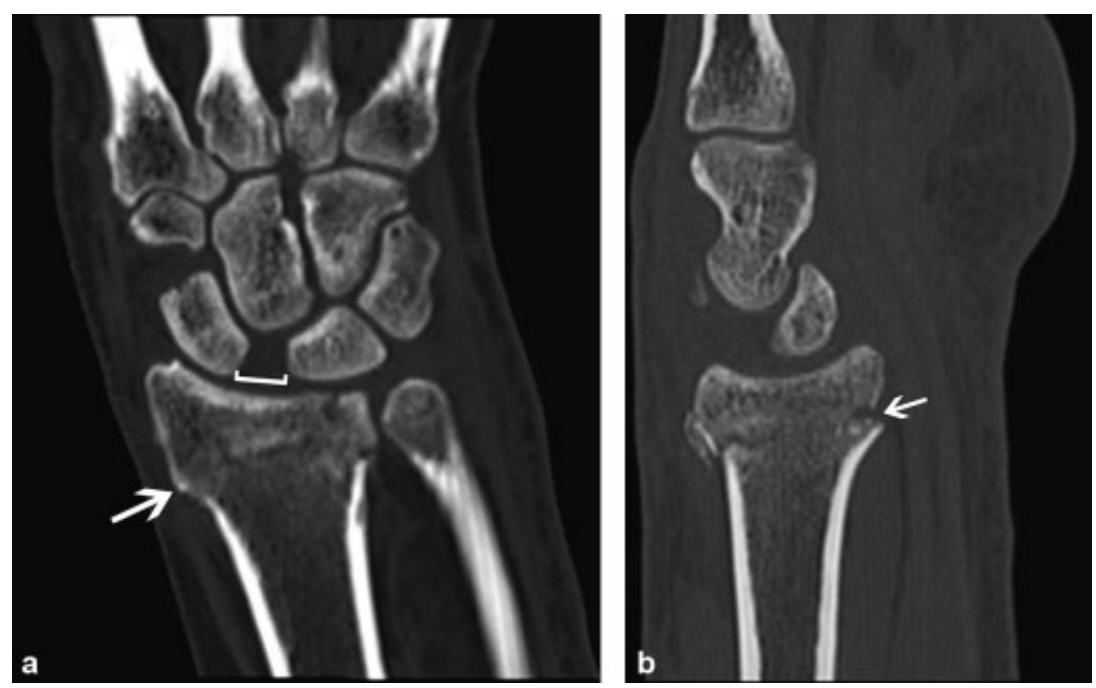

Fig. 4 Wrist fracture and carpal instability. (a) Coronal and (b) sagittal computed tomography images in a 44-year-old man with a backward fall on an outstretched hand demonstrate a comminuted and impacted distal radial fracture (arrows) with dorsal angulation of fracture fragments consistent with a Colles fracture. A concomitant scapholunate dissociation (bracketed) is also demonstrated. 
Ulnar collateral ligament injury of the thumb is rare in the snowboard population, accounting for only $2 \%$ of upper extremity injuries. ${ }^{6}$ These injuries are often related to the use of ski poles that snowboarders do not use.

\section{Mechanism}

Wrist injuries are typically caused by a fall and twice as likely to happen during backward falling compared with falling forward. ${ }^{6,10}$ Moreover, a snowboarder falling on a hand behind the back increases the rate of complex and comminuted fractures. ${ }^{11}$ When a wrist fracture occurs, it is most frequently the trailing arm's wrist that is affected. ${ }^{4}$

Wrist fractures, except the scaphoid, and sprain injuries happen mostly in the beginner group and account for $34 \%$ of all injuries in the beginner group, whereas more experienced snowboarders generally suffer more severe injuries and more scaphoid fractures as well as wrist dislocations. ${ }^{6}$ More specifically, lunate or perilunate fractures and dislocations are also reported in the expert group that are typically a result of a backward fall following an aerial maneuver., ${ }^{4,6}$ Snowboarders wearing wrist guards are half as likely to sustain a wrist injury compared with not wearing wrist guards. ${ }^{6}$

\section{Imaging}

Radiographs, typically including a wrist posteroanterior (PA) view, lateral view, angled PA view with the scaphoid en face, and external oblique view, demonstrate most wrist fractures. However, scaphoid fractures can be radiographically occult in up to $25 \%$ of patients (-Fig. 5). ${ }^{12}$ In addition, fractures of the lunate, hamate, capitate, and triquetrum are also easily overlooked radiographically. ${ }^{13}$

Therefore, patients presenting with a high clinical suspicion of a wrist fracture and a negative initial radiograph should be seen by an orthopaedic surgeon, who can decide to perform additional cross-sectional imaging. Computed tomography (CT) and in some institutions even magnetic resonance imaging (MRI) is readily avail- able, so protective immobilization and unnecessary delay can be avoided. ${ }^{14,15}$ An alternative approach and the historically most commonly used standard is cast immobilization of the wrist with repeat clinical examination and scaphoid-specific radiographs obtained at 2 to 3 weeks after the injury as occult fractures tend to become more apparent. ${ }^{14}$ At the time of follow-up, the occult fracture can be appropriately treated, and ligament injuries can also be further imaged as necessary (power grip wrist radiographs, CT, or MRI). An exception for this last approach might be those for whom immobilization is relatively contraindicated, such as patients with first carpometacarpal arthritis.

$\mathrm{CT}$ is generally performed after the initial radiograph to detect occult wrist fractures and depict the anatomy. However, even CT cannot entirely rule out all wrist fractures. A 2015 Cochrane meta-analysis showed that sensitivity and specificity for CT to detect occult scaphoid fractures were 0.72 (95\% confidence interval [CI], $0.36-0.92)$ and 0.99 (95\% CI, 0.71-1.00), respectively. ${ }^{15}$

MRI has a higher sensitivity (0.88) compared with CT and allows better evaluation of the soft tissues. ${ }^{15}$ However, MRI may not always identify cortical fractures. ${ }^{16}$ Also, MRI is not readily available in every hospital and carries a higher cost. Therefore, CT is the most often used second image modality.

\section{Elbow and Forearm Injuries}

Elbow and forearm injuries are relatively uncommon snowboarding injuries but still occur with a greater frequency than they do in skiers. A study surveying snowboarders in Colorado, including 7,430 snowboard-related injuries over 10 seasons, reported that elbow injuries accounted for $8 \%$ and forearm injuries for $5.5 \%$ of all injuries. ${ }^{6}$ By comparison, only $1 \%$ of alpine ski injuries are elbow injuries. ${ }^{17}$ Another retrospective study of 1,445 injured snowboarders and 10,152 injured skiers observed more elbow dislocations and fractures among snowboarders (30 of 64 cases [47\%])
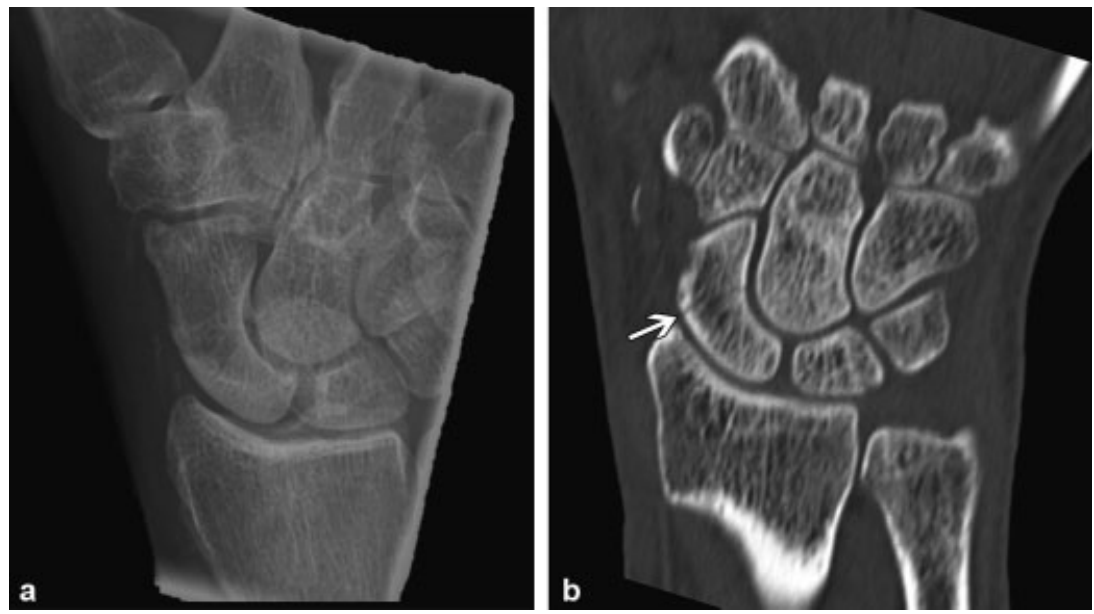

Fig. 5 Hairline fracture of the scaphoid waist. (a) Dedicated posteroanterior radiograph of the scaphoid with ulnar deviation demonstrates subtle lucency in the scaphoid waist. This view is performed as part of a scaphoid series, and ulnar deviation moves the scaphoid away from the radius, rotating it volarly to minimize superimposition of osseous structures. (b) Coronal computed tomography image confirms a hairline fracture (arrow). 
than skiers (26 of 152 cases [17\%]). Furthermore, the dislocation rate with or without elbow fracture was also significantly higher among snowboarders (17 of 64 cases [26.6\%]) than skiers ( 8 of 152 cases [5.3\%]). ${ }^{18}$

Most forearm injuries are fractures (71\%), and of those, combined radius-ulna fractures are relatively more common (57\%). ${ }^{6}$ Monteggia and Galeazzi are rather rare fracture patterns, accounting for only $2 \%$ and $3 \%$ of snowboardrelated forearm injuries, respectively. ${ }^{6}$

\section{Mechanism}

A fall after a jump is a common injury mechanism of an elbow dislocation. The Colorado study reported that $63 \%$ of elbow dislocations resulted from an aerial maneuver. ${ }^{6}$ The direction of the fall does not correlate with the incidence of elbow or forearm injuries. ${ }^{5}$ Elbow dislocations are mostly of the posterior type. ${ }^{18}$

The severity of the elbow injury seems to be mainly the result of a direct force on the elbow that receives the full impact of the fall. This direct injury mechanism is mostly combined with a fall on an outstretched hand, with either elbow extension or a longitudinal thrust force to the proximal radius, ulna, and distal humerus. ${ }^{18}$

\section{Imaging}

Several studies suggest that radiologic imaging is unnecessary if the active range of motion of the elbow is preserved. ${ }^{19}$ Most elbow and forearm fractures can be diagnosed on conventional radiographs using an anteroposterior (AP) and lateral view. The AP view should be obtained with the elbow fully extended and the forearm supinated, allowing optimal visualization of the medial and lateral epicondyles, radiocapitellar joint, and estimation of the carrying angle (angle between the longitudinal axes of the humerus and the forearm on AP projection; normal values: 11 and 13 degrees valgus). ${ }^{20,21}$ For the lateral view, the elbow should be in 90 degrees of flexion and the forearm in the neutral (thumb-up) position. ${ }^{21}$

When an elbow fracture is suspected, an additional lateral oblique view (Coyle's view) of the radiocapitellar joint (tube angled 45 degrees cephalically along the humeral shaft) is needed to detect the sometimes subtle radial head/neck fractures that can otherwise be missed because of osseous overlap of the coronoid process. ${ }^{22}$

The posterior and anterior fat pad sign allows the detection of an elbow effusion and is most useful as a predictor of an intra-articular disease process at the elbow in the absence of any radiographically visible bone abnormality. In the setting of trauma, this effusion most likely represents lipohemarthrosis, suggesting a nondisplaced fracture of the radial head in adults or a supracondylar fracture in children. ${ }^{23}$

Both MRI and ultrasonography (US) are well suited to detect any elbow joint soft tissue injuries of the collateral ligaments, tendons, and muscles. However, US assessment will only achieve high accuracy with sufficient operator experience and anatomical knowledge of the elbow joint structures.

\section{Shoulder and Clavicle}

Shoulder injuries are one of the most common snowboardrelated injuries, occurring twice as frequently compared with skiers. ${ }^{11,24} \mathrm{Kim}$ et al studied snowboard and ski injuries between 1988 and 2006 in Vermont and reported that shoulder soft tissue injuries and clavicle fractures accounted for $12 \%$ and $4 \%$ of all reported snowboarding injuries versus $6 \%$ and $2 \%$ of ski injuries, respectively. ${ }^{5}$ Whereas the overall rate of the (mostly anterior) glenohumeral joint dislocations in snowboarding is 2.3 times higher than in skiers, the prevalence of fracture-dislocations (15\% of combined snowboard and ski shoulder dislocations) is higher for skiers compared with snowboarders, $34 \%$ versus $12 \%$, respectively. ${ }^{25}$

The rise of terrain parks with the purpose of performing ground and aerial maneuvers has led to an increased rate of shoulder and clavicle fractures. The Kim et al study showed that $44 \%$ of clavicle fractures occurred while snowboarding in a terrain park, with $34 \%$ linked to jumping and $93 \%$ as a result of a direct impact on the snow surface. ${ }^{5}$ Most (67-70\%) of shoulder dislocations occur in the intermediate- to expertlevel group, which is likely attributed to increased speed, altitude, and complexity of maneuvers in this group. 6,25

A high incidence of recurrent shoulder dislocations is reported, especially for snowboarders $(<25$ years of age) who were young at the time of their first dislocation. ${ }^{24}$

\section{Mechanism}

One of the main mechanisms of shoulder and clavicle fractures and dislocations is a direct impact following a jump. Ogawa et al retrospectively reviewed cases treated in a Japanese hospital between 2004 and 2009, and they found that glenohumeral joint dislocations are significantly more commonly the result of a fall and often happen while engaging the toe side of the snowboard. Also, the leading-side joint is the most often injured shoulder joint. ${ }^{25}$

The most typical pattern for a shoulder dislocation is engagement of the snowboard's toe-side edge, leading to a fall forward and catching the fall with (the leading) hand or a direct impact on the shoulder girdle, resulting in a shoulder dislocation (-Fig. $\mathbf{3}){ }^{25}$

Rotator cuff injury (strain or tear) is also mostly associated with a fall. The suspected injury mechanism is an abduction-external rotation force against the outstretched arm by the slope during a fall. Alternatively, a direct impact can also result in rotator cuff injury and muscle contusion. ${ }^{24}$ Rotator cuff injuries are likely underreported because patients do not look for immediate medical aid as they often experience vague, more variable, or less severe symptoms.

\section{Imaging}

Conventional radiographs detect most shoulder and clavicle fractures or dislocations. The standard radiograph series for the clavicle is a straight AP view and a 30-degree inclined cephalic view. The shoulder is examined radiographically using a (true) AP, scapular Y view, and ideally 
also an axial view because it has a higher diagnostic yield for subluxation and dislocations. ${ }^{26} \mathrm{~A}$ routine axial projection of the shoulder can be technically difficult, however, and painful in the setting of acute trauma because the affected shoulder needs to be abducted with the elbow resting on the detector. For this reason, multiple variations of the standard axillary projection have been developed to minimize or eliminate shoulder abduction, such as the modified trauma axial and Velpeau view. ${ }^{26}$ Radiographic evaluation of the medial clavicle, and especially the sternoclavicular joint, can be challenging. CT is well suited to assess these locations.

Stress weight-bearing radiographic views of the acromioclavicular $(A C)$ joints are no longer routinely performed because the benefit in the acute setting is controversial. However, some authors found a significant increase in the coracoclavicular (CC) distance when weights were applied, sometimes resulting in the upgrading of Rockwood III injuries (both AC and CC ligaments ruptured), potentially changing the therapy from conservative to surgical treatment. ${ }^{27}$ As a result, there is ongoing equipoise about the role of weighted stress views in this population.

In the case of shoulder dislocation, a noncontrast MRI should be performed to further assess the type and extent of soft tissue involvement to help preoperative planning. MR arthrography was shown to be superior in the diagnosis of labral, cartilage, and partial-thickness rotator cuff tears, but it should be kept for problem solving because it is an invasive technique. ${ }^{28}$

Alternatively, a CT arthrogram can be performed and was shown to be comparable with MR arthrography in assessing the labrum, but inferior for diagnosing partialthickness rotator cuff tears, especially bursal-sided tears. CT arthrogram should be reserved for situations where MRI scanning is not possible (e.g., claustrophobia) because it also exposes the patient to radiation. ${ }^{28}$

US can accurately diagnose rotator cuff and proximal biceps tendon tears but is limited in its ability to detect further intra-articular abnormalities.

\section{Lower Extremity Injuries}

The study in 1989 by Pino and Colville reported that snowboarders more frequently experience lower extremity injuries rather than upper extremity injuries. ${ }^{29}$ However, more recent studies in both the pediatric and adult population demonstrated that the upper extremity is now the principal region of injury among snowboarders, with lower extremity injuries less frequent compared with skiing. ${ }^{30,31}$ Several other studies showed similar rates of lower limb injuries in skiing and snowboarding. ${ }^{32}$ In children and adolescents, lower extremity injuries account for $21 \%$ of all medically treated snowboard injuries and between $19 \%$ and $23 \%$ of all injuries reported by the ski patrol. ${ }^{30} \mathrm{~A}$ similar incidence rate is seen in the adult group where lower extremity injuries account for $12.3 \%$ of all snowboarding injuries. ${ }^{33}$

An important difference between skiing and snowboarding is the anatomical distribution of lower leg injuries. Ankle and foot injuries predominate in snowboarding, whereas knee and boot-top injuries predominate in skiing. As compared with skiers, snowboarders have half the rate of knee injuries (22\% versus $44 \%$ ), and those injuries that do occur are typically less severe. ${ }^{7}$ Furthermore, ankle injuries are four times more common in snowboarding and often represent sprain injuries. ${ }^{29,32}$ This difference can most readily be explained by the fact that snowboarders normally wear the less protective soft-shell boots, allowing more ankle mobility, whereas skiers usually wear hard-shell boots protecting the ankle but transferring forces proximally to the tibia and knee. In addition, snowboard bindings have a fixed angle on the board, minimizing valgus stress on the knee, whereas the skier's feet can rotate independently because they have two separate skis.

The forward limb of the snowboarder is most often injured, which can be attributed to unequal weight distribution and turning technique. ${ }^{33}$ Both ankle and knee injuries occur mostly on the left side, $76 \%$ and $71 \%$, respectively, because most snowboarders have a left leading foot. ${ }^{8,29}$

Femoral fractures are rare in snowboarding (Pino and Colville reported no femoral fracture among 110 snowboard injuries). ${ }^{29}$ The general lower speed of snowboarders might be an explanation because this is known to impact the incidence of femoral fractures in alpine skiing. ${ }^{34}$

\section{Ankle Injuries}

Ankle injuries are one of the most prevalent lower extremity injuries associated with snowboarding, accounting for $15 \%$ of all injuries according to one study. ${ }^{9}$ Ankle sprains (6\%) are more frequent than actual fractures of the ankle (3\%). ${ }^{5}$ Also, the ankle is the most commonly fractured site of the lower extremity fractures in snowboarders (53\% of lower extremity fractures). ${ }^{33}$

Ankle injuries are most common in soft-shell boots that are typically worn by snowboarders because they allow more mobility of the ankle joint, compared with rigid hard-shell boots. 32

\section{Mechanism}

A snowboarder's soft-shell boot is fixed to the board, predisposing to ankle inversion, eversion, or hyperplantarflexion when falling backward. ${ }^{29}$ In a similar fashion, forward falling contributes to ankle hyperdorsiflexion, potentially resulting in the injury of the ankle syndesmotic ligaments, Achilles tendon, and gastrocnemius. ${ }^{29}$ This seems to be confirmed by the observation that riders who use ankle supports, either hard-shelled boots or inserts, experience half the number of ankle injuries compared with those who used soft boots.

The most common mechanism of an ankle fracture is supination-external rotation, according to the Lauge-Hansen classification, with supination-external rotation type II the most common. ${ }^{33}$ The ankle of the leading leg tends to be the most commonly injured. ${ }^{33}$

\section{Imaging}

The Ottawa ankle rules (for adults and children $>5$ years) remain valid to determine the need for ankle 
radiographs. ${ }^{35}$ A routine ankle radiograph series includes an AP view, true lateral view, and a mortise view (leg rotated internally 15 degrees, thus aligning the intermalleolar line parallel to the detector). ${ }^{36}$

In addition to a clinical examination (including special tests, such as the external rotation test), stress weightbearing radiographs (if tolerated by the patient) may assist in detecting latent tibiofibular syndesmotic injury. ${ }^{37}$ Syndesmotic injuries, also known as high ankle sprains, can be challenging to diagnose on static radiographs when the fibula remains reduced with absent tibiofibular joint space widening. Especially grade II syndesmotic injuries can be occultly unstable, and if left untreated, they can result in chronic instability, pain, and osteoarthritis. ${ }^{38}$

When an ankle effusion is detected on the lateral view, a CT view should be performed to detect a potential occult fracture. In one series, $33 \%$ of patients with an ankle effusion but no detectable fracture on radiography had an occult fracture confirmed on $\mathrm{CT}^{39}{ }^{39}$ which otherwise is not indicated for primary imaging of an ankle injury but may assist and better characterize a fracture and may assess soft tissues. ${ }^{40}$

MRI is particularly useful in the setting of suspected high sprain injury with a sensitivity of $100 \%$ and specificity of 70 to $100 \%{ }^{41}$ MRI has a superior soft tissue contrast resolution and will show either disruption or a wavy appearance of the anterior inferior tibiofibular ligament and the posterior inferior tibiofibular ligament. Indirect signs are fluid extending within the tibiofibular space and prolapse of interspace fat between the anterior and posterior tibiofibular ligaments (-Fig. 6). ${ }^{42}$

MRI detects lateral ligaments tears with a sensitivity of 75 to $100 \% .{ }^{43}$ However, MRI should not be routinely performed in this setting because findings do not correlate with clinical outcome, and most ligamentous injuries heal with sufficient strength to maintain joint stability with conservative management. ${ }^{40,43}$
US is a useful and sensitive examination of the ankle joint ligaments in the hands of an experienced radiologist but generally less accurate compared with MRI. ${ }^{44}$

Lateral Talar Process Fractures (Snowboarder's Fracture)

A lateral talar process fracture is an uncommon injury but very specific to snowboarding. The reported incidence ranges between $1.2 \%$ and $6.3 \%$ of all lower extremity injuries. $^{45}$ The clinical importance of this lesion is that it can easily be misdiagnosed as a severe ankle sprain. If this fracture remains untreated, it can lead to nonunion, talar osteonecrosis, and accelerated subtalar arthrosis. ${ }^{46}$

\section{Mechanism}

This type of injury typically occurs as a result of forceful axial loading (e.g., landing after an aerial maneuver). The axial loading of the ankle is combined with forced ankle dorsiflexion and ankle inversion, leading to the fracture of the lateral talar process and cartilage damage. ${ }^{9,45}$ However, a more recent experimental cadaveric study (2003) found that ankle dorsiflexion combined with eversion, rather than inversion, most likely produced lateral talar process fractures (- Fig. 7 ). ${ }^{47}$

\section{Imaging}

The fracture is often difficult to identify on plain film, especially when it is small and nondisplaced. Although standard radiographs can show the lateral process fracture, up to $50 \%$ are missed. ${ }^{46}$ Therefore, if there is a high clinical suspicion, CT is preferred (-Fig. 8).

MRI typically shows focal bone marrow edema in the region of the lateral talar process. A hypointense fracture line can be seen but will not always be appreciable, making differentiating bone contusion or subtalar impingement difficult. Therefore, $\mathrm{CT}$ is preferred to allow the detection of subtle talar process fractures seen as a fracture line or cortical step-off.

The three types are as follows: type 1 is a chip fracture of the articular process of the talus that does not extend to
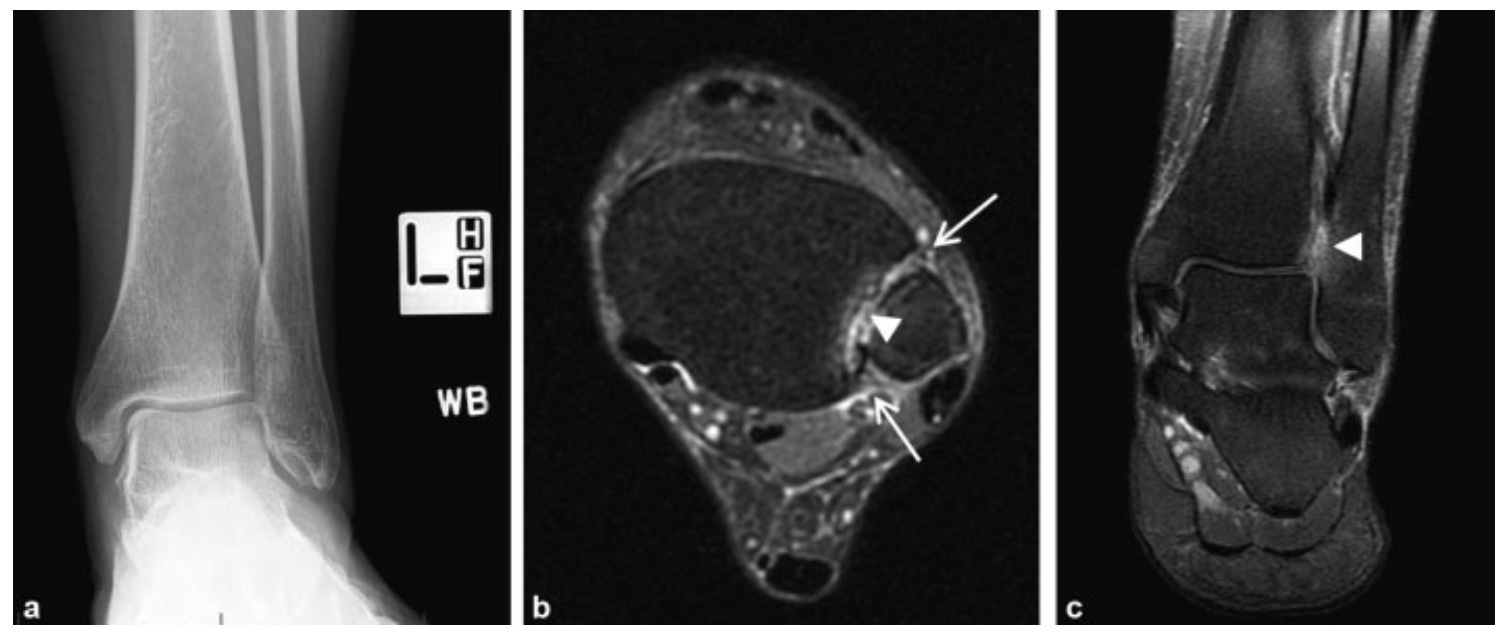

Fig. 6 High ankle sprain injury in a 40-year-old snowboarder caused by a forward fall. (a) Normal weight-bearing radiographic anteroposterior view shows no widening of the tibiofibular joint space. (b) Axial proton-density fat-saturated sequence shows disruption of the anterior and posterior tibiofibular ligaments (arrows) and fluid extending in the tibiofibular joint space (arrowhead), as also seen on (c) the coronal image. 


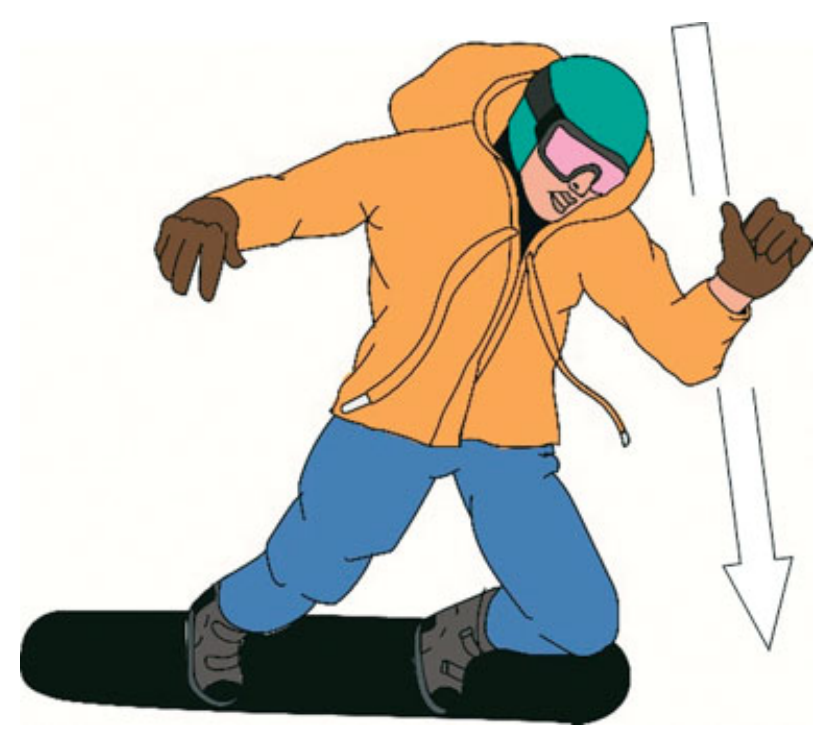

Fig. 7 Injury mechanism of a lateral talar process fracture (snowboarder's fracture). The snowboarder performed a jump and falls straight down, resulting in a forceful axial load on his leading left ankle that is dorsiflexed and everted at the time of impact.

the talofibular joint; type 2 is a single large fragment extending from the talofibular to the subtalar joint; and type 3 is a comminuted fracture involving the entire lateral process. ${ }^{48}$

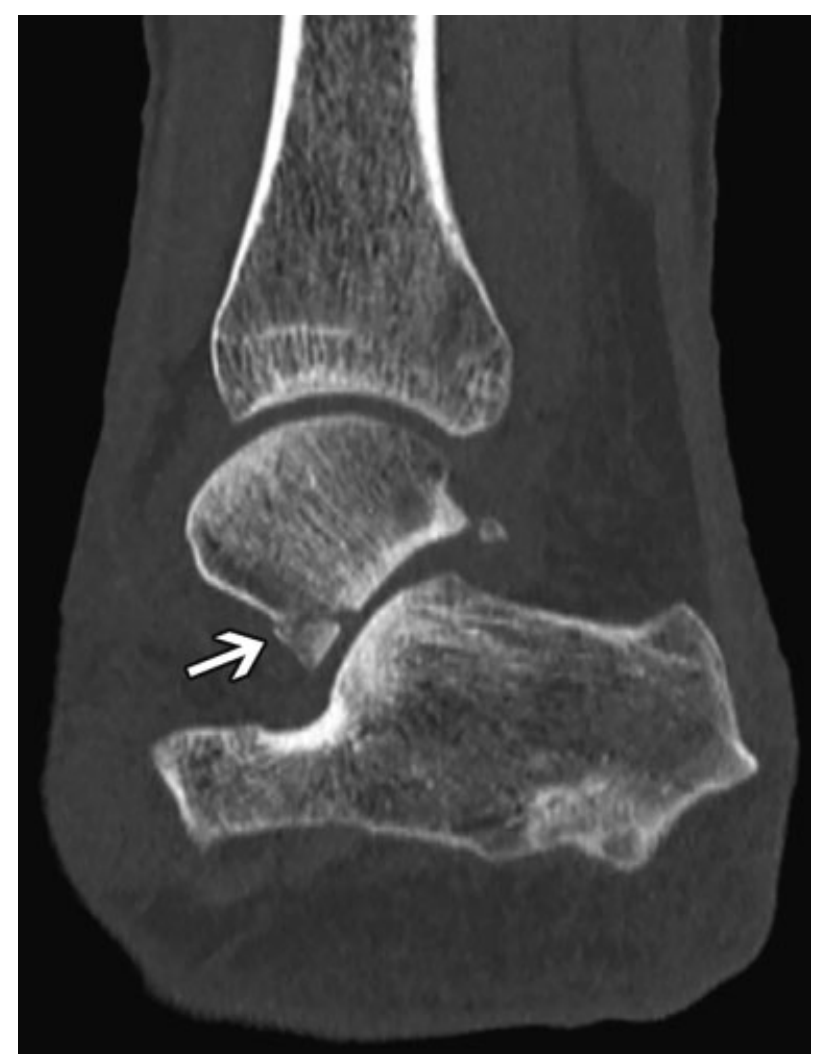

Fig. 8 Snowboarder's fracture. Sagittal computed tomography image of a 31-year-old woman who sustained a traumatic fall demonstrates a nondisplaced intra-articular fracture of the lateral talar process (arrow), consistent with a snowboarder's fracture.

\section{Chronic Boot-Related Injuries}

Malleolar bursitis and so-called pseudotumor of the ankle are similar but rare lesions that are the result of prolonged abnormal contact pressure and shear forces that arise between the bony malleoli and the snowboarder's boot. Clinically, these lesions present as asymptomatic or tender lateral or medial malleolar soft tissue swelling that can be fluctuant in case of an adventitial bursa. ${ }^{49}$

\section{Mechanism}

The malleolar regions normally do not have anatomical bursae, but a newly developed, or adventitia, subcutaneous bursa can arise adjacent to the malleolus. The bony prominences of the malleoli stick out and have little inherent soft tissue to cushion from excessive pressure and friction. Abnormal friction and pressure caused by the (typical new or ill-fitting) snowboard boot can potentially lead to fat necrosis and local inflammatory changes. ${ }^{50}$ If activity persists, this can eventually lead to the formation of an adventitial bursa, which is a suboptimal anatomical cushion. ${ }^{49}$ These adventitial bursae have a thick fibrous wall, compared with normal bursae, and they are more prone to inflammation that can lead to so-called malleolar bursitis. $^{49}$

A pseudotumor of the ankle has a similar etiology. It is the result of chronic friction caused by the boot but lacks the formation of a bursa. It occurs more often in the supramalleolar and lateral regions. ${ }^{51}$ Chronic compression probably leads to fat necrosis, subcutaneous inflammation, and fibrosis, resulting in a pseudotumor or masslike lesion. ${ }^{50}$

\section{Imaging}

Plain film demonstrates soft tissue swelling overlying the malleolus with no underlying bone abnormalities or soft tissue calcifications. Cortical thickening of the distal fibula was described in two professional snowboarders. ${ }^{50}$

The adventitial malleolar bursa can easily be demonstrated by US and MRI that will show a fluid-containing cystic structure with or without septations and/or complex content. MRI typically shows a discrete mass with well-defined margins and fluid characteristics on all pulse sequences. ${ }^{52}$ In addition to the fluid signal characteristics, areas of low-signal-intensity stranding can be present on both T1-weighted and T2-weighted images, representing scarring of a bursa, which is possibly secondary to prior bursal inflammation or bleeding. ${ }^{52}$

MRI findings of a malleolar pseudotumor are ill-defined soft tissue swelling over the (typically lateral) malleolus, loss of normal T1 hyperintensity of the subcutaneous fat tissue, and prolonged T2 relaxation, as well as contrast enhancement. Adjacent reactive periosteal edema of the fibula can be present (-Fig. 9). ${ }^{50}$

\section{Knee}

Knee injuries are among the most common winter sports injuries, especially in skiing, whereas snowboarders suffer significantly fewer knee injuries. A 4-year prospective study 


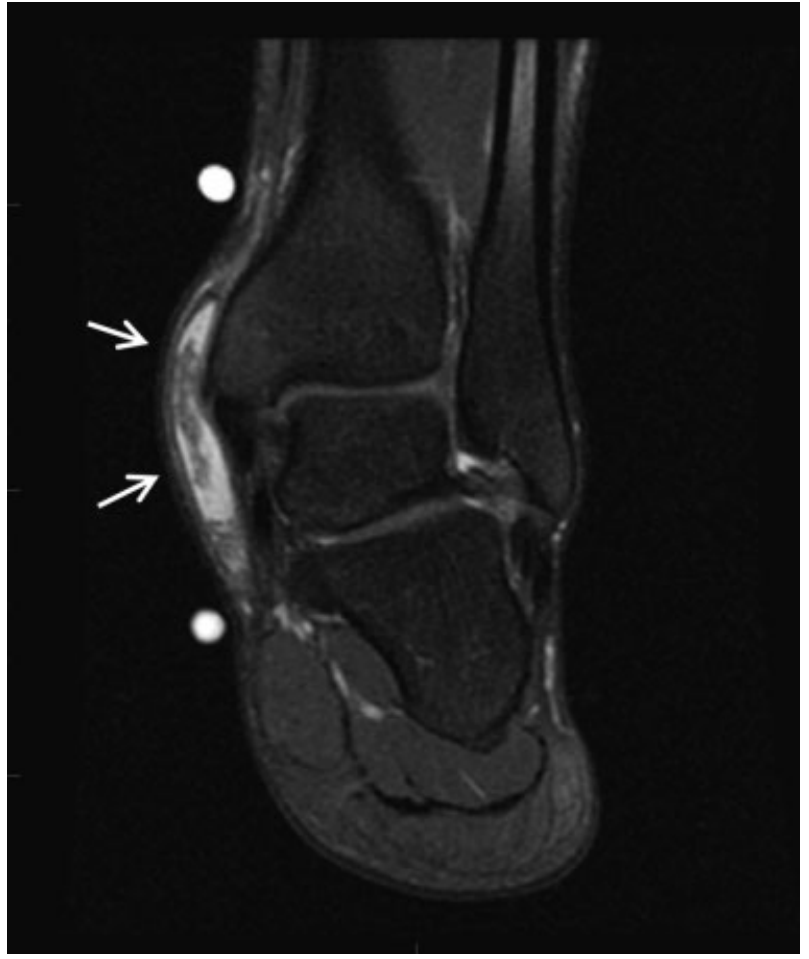

Fig. 9 Lateral malleolar bursa. Coronal proton-density-weighted fatsuppressed magnetic resonance image in a 16-year-old professional snowboarder with fluctuating medial ankle mass shows a complex fluid collection superficial to the medial malleolus (arrows) indicative of an adventitious medial malleolar bursa. The combination of fat necrosis, edema, and fibrosis produces the complex appearance that potentially mimics other tumors and pseudotumors.

at three Australian ski resorts showed that knee injuries occurred half as frequently ( $23 \%$ versus $44 \%$ ) in the snowboarders' group compared with skiers. ${ }^{32}$ Moreover, snowboarders tend to have less severe knee injuries (grade I) compared with skiers (grades II and III). ${ }^{32}$

Snowboarders rarely sustain ACL injuries compared with skiers. But snowboarders suffer MCL injuries more frequently rather than $A C L$ injuries (4\% versus $2.5 \%$ ). ${ }^{5}$ A 2008 survey found that only $1 \%$ of the injuries were ACL ruptures compared with $12.6 \%$ in alpine skiing. ${ }^{53}$ A study of $21,303 \mathrm{ACL}$ ruptures from French ski resorts demonstrated minimal numbers of snowboarding ACL injuries. ${ }^{53}$ However, because snowboarders tend to seek more thrills with higher and bigger jumps, especially in snow parks, the rate of ACL injuries is increasing. ${ }^{54}$

Novice snowboarders and those wearing hard-shell boots are more likely to sustain a knee injury. ${ }^{32}$ Additionally, expert snowboarders have a relatively higher proportion of knee injuries (17\% of all acute injuries) compared with recreational snowboarders, ${ }^{55}$ and they are more likely to sustain an ACL rupture, which is most likely the result of an increased frequency, complexity, and velocity of aerial maneuvers. ${ }^{56}$

\section{Mechanism}

Similar to upper extremity injuries, the leading knee is more prone to injury. ${ }^{5}$ Contrary to skiers, snowboarders have non-release bindings, meaning the boots remain fixed to the board and do not release when an external force is applied to the boot (e.g., fall or tumbling). These snowboard non-release bindings decrease the risk of high torque forces and provide rotational stability to the knees. Therefore, knee injuries occur less often in the snowboarding population, whereas skis are able to move independently, allowing both rotational and valgus forces to the knees. ${ }^{57}$ This effect is likely reduced in elite snowboarders because the impact and rotational forces are higher with increasingly spectacular jumps.

Multiple studies showed that jumping promotes knee injuries in both recreational and professional snowboarders. ${ }^{55}$ In accordance, ACL ruptures are shown to mostly occur when a snowboarder hits a flat surface after jumping. ${ }^{56} \mathrm{~A}$ proposed mechanism for the ACL rupture is an eccentric contraction of the quadriceps muscles when landing from a height with the knee slightly flexed. The resulting anterior tibial translation, valgus, and internal tibial rotation can exert enough strain on the ACL to rupture it. ${ }^{56}$

The stance of the snowboarder can also contribute to ACL injury. The snowboarder's leading foot binding is usually positioned in slight external rotation. While riding, the body is mostly facing forward/in the direction of downhill sliding, more so than the actual angulation of the front-foot binding, resulting in actual internal tibial rotation at the knee (-Fig. 10). Internal tibial torque applied to a fully extended knee is proven to be the most dangerous loading condition for ACL injury. ${ }^{56}$

\section{Imaging}

Detailed history, physical examination, and radiographs, including an AP view and lateral view, provide an accurate diagnosis in $83 \%$ of traumatic knee cases. ${ }^{58}$ The supine

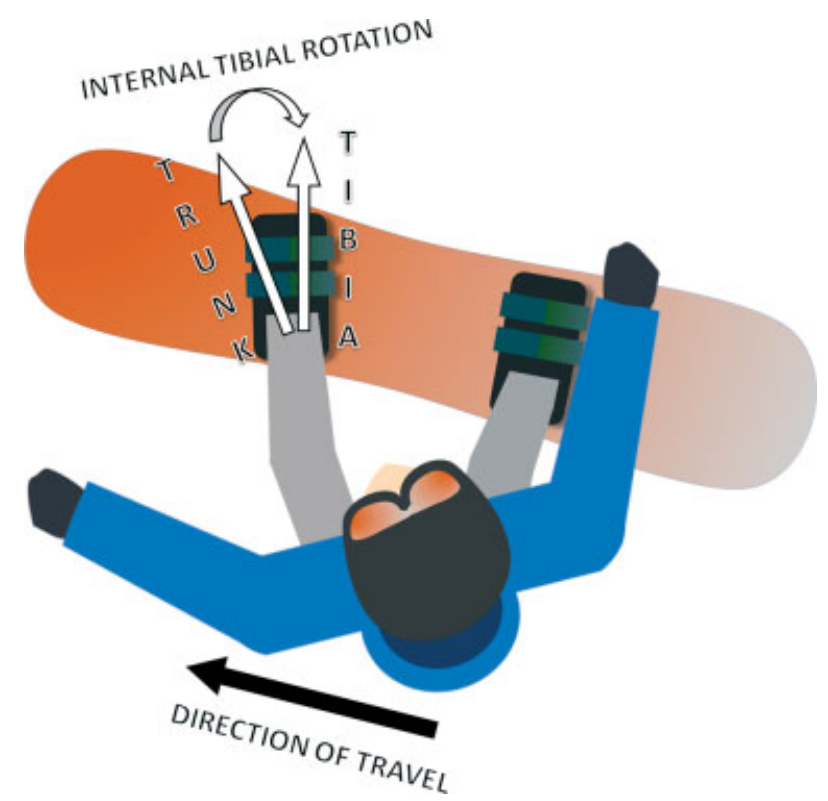

Fig. 10 Typical stance in snowboarding with slight external rotation of the front foot and the torso facing frontward, resulting in actual internal rotation of the tibia. 
lateral horizontal beam view is highly sensitive for a knee joint effusion, which is a secondary sign of posttraumatic knee joint derangement or other intra-articular diseases (e.g., infection and arthritis). ${ }^{59}$

The Ottawa knee rules ( $>55$ years old; inability to weight-bear immediately after trauma and in the emergency department (ED) for four steps; isolated patellar tenderness, fibular head tenderness; inability to flex the knee to 90 degrees) can be useful in clinical practice to effectively rule out knee fractures and prevent unnecessary radiographs. ${ }^{60}$ However, if there is clinical evidence of an effusion/hemarthrosis, even when the patient is still able to weight-bear, radiographs should be performed to rule out any (intra-articular) fractures.

Because knee radiographs have a low yield of showing fractures, CT can be used as the secondary imaging modality to assess for possible occult fractures. ${ }^{61}$ In one study, CT had a sensitivity of $100 \%$ and plain film $83 \%$ in detecting tibial plateau fractures, and CT was superior to characterize the fracture severity and type. ${ }^{62}$

MRI has a high accuracy in detecting both meniscal and ligamentous injuries (-Fig. 11). ${ }^{63}$ Fluid-sensitive sequences (proton-density fat-saturated and T2 fat-saturated) depict ligamentous injury as well as bone contusion (bone marrow edema). ${ }^{64}$ In contrast, T1-weighted sequences are well suited to visualize fracture lines. Therefore, MRI should be used to aid in the diagnosis and further characterization of bone and soft tissue injuries of the knee. ${ }^{61} \mathrm{~A}$ 1.5-T MRI scanner is sufficient because 3-T imaging has not proven to result in higher accuracy in detection of meniscal and ACL injuries. ${ }^{65}$

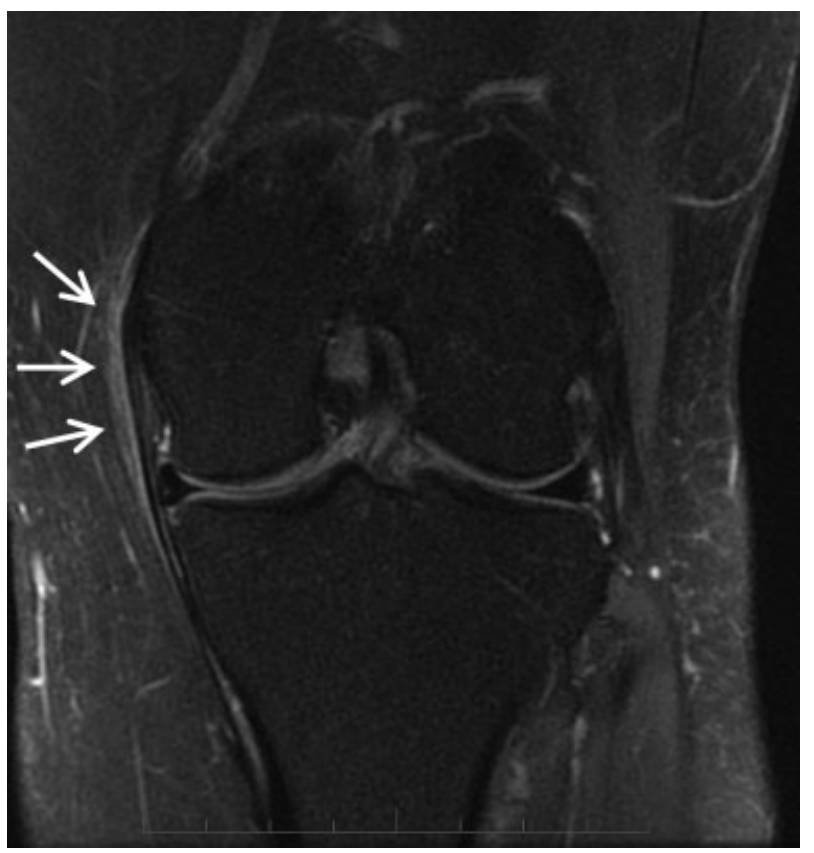

Fig. 11 Severe sprain injury of the medial collateral ligament (MCL) in a 35-year-old snowboarder following a twisting injury of the knee after a fall. Coronal proton-density fat-saturated magnetic resonance imaging sequence demonstrates soft tissue edema superficial to the otherwise grossly preserved MCL (arrows).
US is not used routinely in the setting of acute knee trauma. ${ }^{61}$ US does allow evaluation of a joint effusion/lipohemarthrosis and the integrity of the collateral ligaments, distal hamstring, and quadriceps tendons. However, US is limited because it does not allow accurate assessment of the cruciate ligament, cartilage, and meniscus.

\section{Pelvis and Sacrum}

About $2 \%$ of all snowboarding-related fractures, or an incidence of 0.102 per 10,000 ski lift tickets, are pelvic fractures according to Ogawa et al, who studied snowboarding injuries over a period of 8 years. ${ }^{66}$ Pelvic fractures were the fifth highest rate of all fractures and mainly consisted of isolated pubic bone (most often superior and/or inferior rami) and/or ischium fractures (46.9\%), followed by isolated sacral fractures (24.1\%). ${ }^{66}$ All cases of a pelvic fracture involving multiple fracture sites also had a sacral fracture. ${ }^{66}$ It is important to note that isolated sacral fractures are infrequent in the general population ${ }^{67}$ but fairly common in the snowboarding population. Therefore, a sacral fracture should be included in the differential diagnosis of any snowboarder with posttraumatic buttock pain.

Pelvic fractures are mostly sustained by intermediatelevel snowboarders (57\%) and to a lesser extent by professionally trained snowboarders $(10 \%) .{ }^{66}$

\section{Mechanism}

Jumping is the main mechanism of pelvic fractures ( $80 \%$ of cases), whereas a collision (with trees, ski tower, or other slope participants) tends to be more common in the group of unstable pelvic fractures. ${ }^{66}$

In activities different from snowboarding, pelvic fractures are mostly the consequence of high-energy/highvelocity impact and therefore often associated with concurrent traumatic injury elsewhere in the body. ${ }^{68}$ In snowboarding, pelvic fractures are not generally associated with high-velocity trauma because only $19.3 \%$ of snowboarders indicate their self-estimated sliding speed as "fast" at the time of injury. ${ }^{66}$

A significant portion (21\%) of snowboarders with pelvic fractures have injuries to other body parts (concussions, face fracture, abdominal injury, spine fractures, etc.). ${ }^{66}$ Unstable pelvic fractures are an even higher percentage (33\%) of concomitant injuries. ${ }^{66}$

Most snowboard-related pelvic fractures (85\%) are stable (type A according to the Tile classification), and $14.5 \%$ are unstable fractures (Tile type $\mathrm{B} / \mathrm{C}$ ). ${ }^{66}$

Contrary to the proven protective effects of wrist guards, wearing shock-absorbing hip pads does not seem to effectively reduce the number of pelvic fractures. ${ }^{66}$

\section{Imaging}

If a pelvic fracture is suspected, a bedside AP radiograph of the pelvis should be performed first (-Fig. 12). An initial radiographic evaluation allows the detection of most clinically relevant pelvic fractures. ${ }^{69}$ Additional radiographic views that may be helpful and can be acquired without 


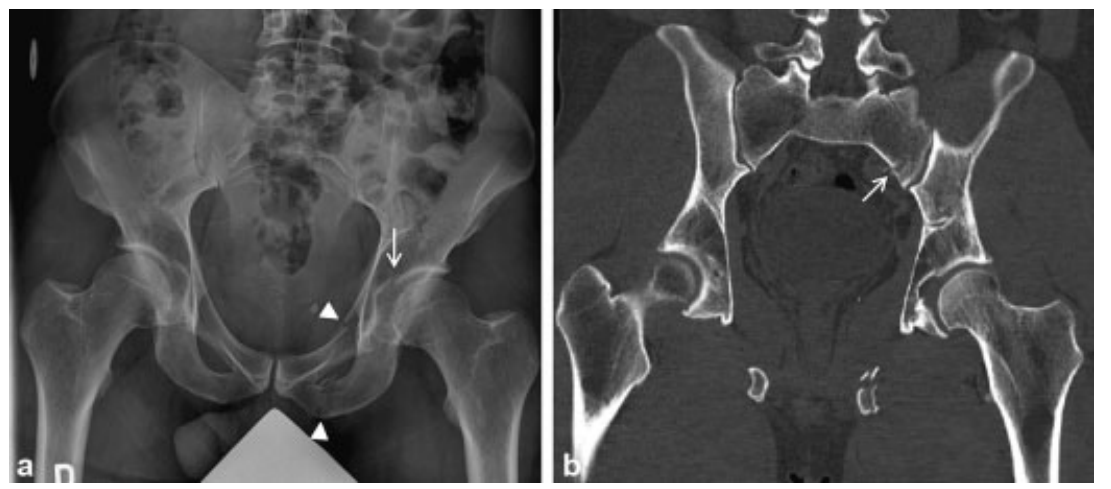

Fig. 12 High-speed trauma while snowboarding with fall on left hip. (a) Anteroposterior view radiograph demonstrates fractures of the left superior acetabulum (arrow) and superior/inferior pubic rami (arrowheads). (b) Coronal computed tomography bone-window image also shows a nondisplaced fracture through the left sacrum that was hardly visible on the radiograph.

moving the patient are the pelvic outlet and inlet views to better depict sacroiliac joints, sacrum, and cranial or caudad displacement of fracture fragments. ${ }^{70}$ Judet views can also be obtained to better depict associated acetabular fractures and are often requested by orthopaedic surgeons for surgical planning because they are easily reproducible in the operating room. ${ }^{70}$

CT has become routine for further work-up of pelvic injury because it provides a better depiction and characterization of the true extent of pelvic fractures and shows potential associated soft tissue injuries. If the patient is hemodynamically unstable, $\mathrm{CT}$ angiography is necessary to detect active bleeding that might need urgent embolization. ${ }^{70}$

Sacral fractures can be especially hard to identify on plain film because of significant soft tissue overlay (-Fig. 12). Therefore, the threshold to perform CT to exclude sacral fracture in case of a snowboarder with buttock pain should be low.

MRI is rarely indicated in the setting of acute pelvic trauma, but it can provide additional information on ligamentous injury. The longer acquisition times and limited availability makes MRI impractical in an acute trauma setting. ${ }^{70}$

\section{Head and Spine Injuries}

\section{Head}

A recent study evaluating snowboarders admitted to the ED of a Swiss alpine trauma center found that a significant portion $(17 \%)$ of injured snowboarders suffer traumatic brain injury (TBI) with most described as mild TBI (92\%). ${ }^{71}$ TBI can be fatal in both snowboarding and skiing and contributes to 43 to $88 \%$ of all injury-related deaths. ${ }^{72}$

Xiang et al analyzed 77,300 ski and 62,000 snowboard injuries treated in U.S. EDs and found an even distribution of head and face injuries among skiers and snowboarders, accounting for $\sim 16 \%$ of total injuries. ${ }^{73}$

Head injury is the most common cause of morbidity and death in snowboarders. ${ }^{34}$ Clinical signs of concussions are present in $22 \%$ of head injuries. ${ }^{55}$ An acute subdural hematoma is the most common head injury that requires surgical intervention (-Fig. 13). ${ }^{3}$
Beginners are more likely to sustain head injuries (34-51\% of cases) compared with intermediate (16\%) or expert snowboarders $(11 \%)^{74}$

\section{Mechanism}

Most severe head injuries are the result of a simple fall on the ski slope (58\% of cases), typically on the occiput. ${ }^{74}$ Falling while jumping and colliding with other people or obstacles are both the cause of $21 \%$ of head injuries. ${ }^{74}$

Snowboarders have their feet fixed to the snowboard and slide sideways, which results in anteroposterior instability. Snowboarders tend to fall backward (68\% of head injuries), typically when opposite-edging occurs

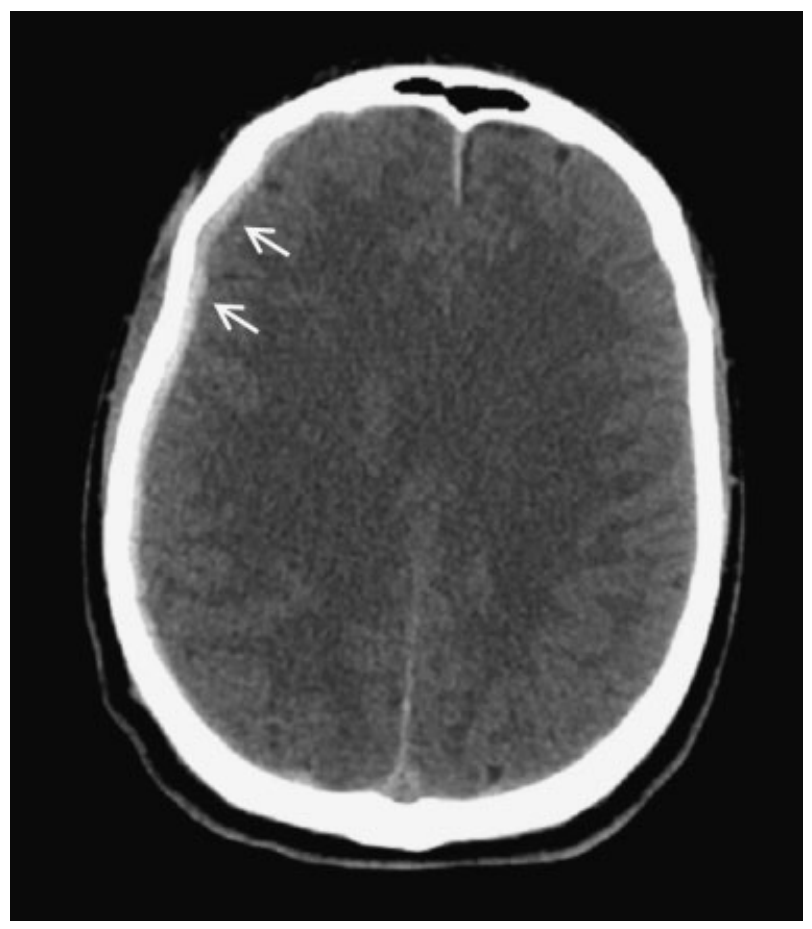

Fig. 13 A 24-year-old snowboarder fell in uncertain circumstances and was found with a decreased level of consciousness. After conservative treatment, the patient was discharged 2 days after the injury. Axial computed tomography image shows right-sided shallow frontal to parietal subdural hematoma (arrows). 
during downhill sliding (facing the mountain) while supporting on their front-side edge ( $\mathbf{- F i g . 1 )} .{ }^{74}$ Only one edge of the board should be touching the snow surface, but when the wrong edge unintentionally hits the snow (opposite-edging), the snowboarder's gravity will suddenly shift to the opposite way. The occiput is thus propelled into the ground (or any intervening objects) behind the snowboarder. The opposite-edging phenomenon happens mostly on gentle rather than steep slopes because the valley-side edge of the board is more likely to hit the snow surface. ${ }^{74}$

The evidence clearly supports the use of helmets because it decreases both the risk and severity of head injury. ${ }^{72}$ Luckily, helmet usage has been progressively increasing over the years. Data from the 2012-13 National Demographic Study of the National Ski Areas Association, encompassing $>130,000$ interviews across the United States, showed that $71 \%$ of skiers and snowboarders wore a helmet, up from $67 \%$ in $2011-12,57 \%$ in $2009-10$, and only $25 \%$ in $2002-3$. $^{75}$

Despite early concerns that helmets may elicit riskseeking behavior or increase the risk of cervical spine injury, the beneficial effects of helmets persist as compared with non-helmeted participants in both skiing and snowboarding. ${ }^{72}$

\section{Imaging}

CT is the imaging modality of choice for the initial assessment because it is readily available, quick, and ideal to detect TBIs and fractures that need urgent surgical intervention (e.g., hemorrhage with mass effect). However, CT is often unable to show the total extent of brain damage because it is insensitive to diffuse axonal injury. ${ }^{76}$ In patients with unexplained neurologic symptoms and (near) normal CT findings, MRI may be used to assess for possible diffuse axonal injury. $\mathrm{T} 2{ }^{*}$-weighted imaging, especially susceptibility-weighted imaging, is highly sensitive to hemorrhagic traumatic axonal injury (TAI), whereas fluid-attenuated inversion recovery allows detection of nonhemorrhagic TAI. ${ }^{77}$

\section{Spine Injuries}

Neurologic injuries, more specifically head and spine injuries, are the leading cause of (mostly permanent) disability and death in both skiing and snowboarding, despite representing only a small portion of overall injuries. ${ }^{78,79}$ Trauma to the axial skeleton comprises 1 to $17 \%$ of all reported ski and snowboard injuries. ${ }^{3}$

\section{Mechanism}

Spine injuries are more often the result of an aerial maneuver, rather than a collision. ${ }^{3}$ The thoracolumbar spine is the most commonly affected level in both snowboarding and skiing. ${ }^{3}$

A burst fracture with anterior dislocation of the flexion-distraction type is one of the most common injury patterns (-Fig. 14). ${ }^{79}$ A proposed mechanism of this type of fracture is a backward fall after an intentional jump. ${ }^{80}$ The snowboarder jumps, loses control while becoming airborne, pitching tailward, and lands on a hard surface in an uncontrolled manner resulting in an axial load (e.g., landing on feet or rear) and forward flexion.

Cervical spine injuries in snowboarding are less common and also mostly less severe, compared with injuries to the thoracolumbar junction that often lead to more severe neurologic deficits. ${ }^{79,81,82}$ It is believed that one of the most common injury patterns of cervical spine fractures is hyperflexion and hyperextension after falling.

\section{Imaging}

Conventional radiography is generally reserved for evaluation of the cervical and thoracolumbar spine with a low suspicion of injury. ${ }^{83}$ Patients with a moderate to high risk of spine injury and/or severe multitrauma should be primarily investigated with $\mathrm{CT}$ due to the high risk of associated injuries. ${ }^{83}$

For the thoracolumbar level, CT is a better and more sensitive examination than radiography to detect spine fractures, especially small cortical fractures. ${ }^{83}$ It is also of

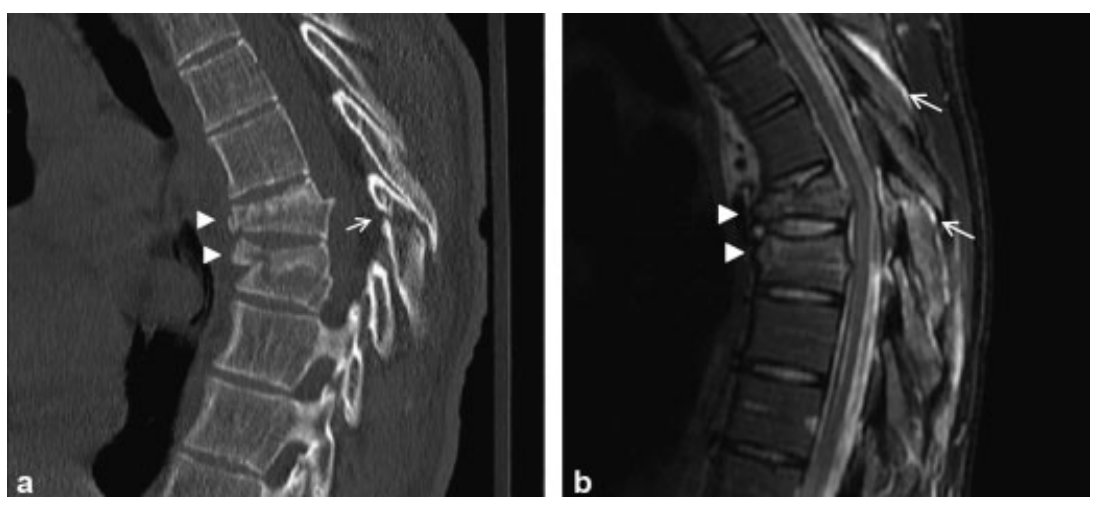

Fig. 14 High-speed injury after hitting a snow boulder, resulting in a thoracic spine flexion-distraction injury with burst fractures. (a) Sagittal computed tomography image shows burst fractures of vertebral bodies T7 and T8 (arrowheads) with anteriorly displaced fracture fragments and fracture of the lamina and spinous process (arrow). (b) Sagittal magnetic resonance imaging (short tau inversion recovery) image depicts interspinous ligament tears (arrows). Mild retropulsion of the T7 and T8 posterior walls is noted, resulting in mild central canal narrowing. 
utmost importance to assess the entire spine on visceral organ-targeted CT performed in the setting of blunt trauma. The thin-slice multiplanar reformats are sufficiently sensitive to detect spine fractures. ${ }^{83}$

CT serves to evaluate the bony structures, whereas MRI is essential to evaluate the spine-related soft tissues (ligaments, spinal cord, nerve roots, muscles, and disks) and to detect occult spinal cord injury when there is a discordance between the clinical status and CT imaging. ${ }^{83}$

\section{Conclusion}

Snowboarding has become one of the most popular winter sports and is associated with an increasing rate of MSK injuries, especially since the advent of terrain parks. Novice snowboarders are most susceptible to injury, whereas expert riders generally sustain a more severe injury. Compared with skiing, snowboarding has a higher injury rate, and the wrist is by far the most injured body part. Future developments should continue promoting the use of protective gear (e.g., wrist guards and helmets) and educational prevention programs.

\section{Conflict of Interest}

None declared.

\section{References}

1 Matsumoto K, Miyamoto K, Sumi H, Sumi Y, Shimizu K. Upper extremity injuries in snowboarding and skiing: a comparative study. Clin J Sport Med 2002;12(06):354-359

2 Vanat L. 2020 International Report on Snow \& Mountain Tourism. 2020. Date of access: Feb. 1st, 2021 at: https://www.vanat.ch/RMworld-report-2020.pdf

3 Owens BD, Nacca C, Harris AP, Feller RJ. Comprehensive review of skiing and snowboarding injuries. J Am Acad Orthop Surg 2018; 26(01):e1-e10

4 Quinlan NJ, Patton CM, Johnson RJ, Beynnon BD, Shafritz AB. Wrist fractures in skiers and snowboarders: incidence, severity, and risk factors over 40 seasons. J Hand Surg Am 2020;45(11): 1037-1046

5 Kim S, Endres NK, Johnson RJ, Ettlinger CF, Shealy JE. Snowboarding injuries: trends over time and comparisons with alpine skiing injuries. Am J Sports Med 2012;40(04):770-776

6 Idzikowski JR, Janes PC, Abbott PJ. Upper extremity snowboarding injuries. Ten-year results from the Colorado snowboard injury survey. Am J Sports Med 2000;28(06):825-832

7 Sasaki K, Takagi M, Kiyoshige Y, Ogino T. Snowboarder's wrist: its severity compared with Alpine skiing. J Trauma 1999;46(06): 1059-1061

8 Abu-Laban RB. Snowboarding injuries: an analysis and comparison with alpine skiing injuries. CMAJ 1991;145(09):1097-1103

9 Sachtleben TR. Snowboarding injuries. Curr Sports Med Rep 2011; 10(06):340-344

10 Sutherland AG, Holmes JD, Myers S. Differing injury patterns in snowboarding and alpine skiing. Injury 1996;27(06):423-425

11 Dohjima T, Sumi Y, Ohno T, Sumi H, Shimizu K. The dangers of snowboarding: a 9-year prospective comparison of snowboarding and skiing injuries. Acta Orthop Scand 2001;72(06):657-660

12 Jenkins PJ, Slade K, Huntley JS, Robinson CM. A comparative analysis of the accuracy, diagnostic uncertainty and cost of imaging modalities in suspected scaphoid fractures. Injury 2008;39(07):768-774
13 Welling RD, Jacobson JA, Jamadar DA, Chong S, Caoili EM, Jebson PJL. MDCT and radiography of wrist fractures: radiographic sensitivity and fracture patterns. AJR Am J Roentgenol 2008; 190(01):10-16

14 Yin Z-G, Zhang J-B, Kan S-L, Wang X-G. Diagnosing suspected scaphoid fractures: a systematic review and meta-analysis. Clin Orthop Relat Res 2010;468(03):723-734

15 Mallee WH, Wang J, Poolman RW, et al. Computed tomography versus magnetic resonance imaging versus bone scintigraphy for clinically suspected scaphoid fractures in patients with negative plain radiographs. Cochrane Database Syst Rev 2015;(06): CD010023

16 Memarsadeghi M, Breitenseher MJ, Schaefer-Prokop C, et al. Occult scaphoid fractures: comparison of multidetector CT and MR imaging-initial experience. Radiology 2006;240(01): 169-176

17 Molinari M, Bertoldi L, Zucco P. Epidemiology of skiing injuries in a large Italian ski resort during 1988-1992. In: Mote C, Johnson R, Hauser W, Schaff P, eds. Skiing Trauma and Safety: Tenth Volume. Conshohocken, PA: ASTM International; 1996:87-97

18 Takagi M, Sasaki K, Kiyoshige Y, Ida H, Ogino T. Fracture and dislocation of snowboarder's elbow. J Trauma 1999;47(01): 77-81

19 Darracq MA, Vinson DR, Panacek EA. Preservation of active range of motion after acute elbow trauma predicts absence of elbow fracture. Am J Emerg Med 2008;26(07):779-782

20 Cain EL Jr, Dugas JR, Wolf RS, Andrews JR. Elbow injuries in throwing athletes: a current concepts review. Am J Sports Med 2003;31(04):621-635

21 Morrey BF. The Elbow and Its Disorders. 3rd ed. Philadelphia, PA: Elsevier Health Sciences; 2009

22 Greenspan A, Norman A. The radial head, capitellum view: useful technique in elbow trauma. AJR Am J Roentgenol 1982;138(06): 1186-1188

23 Goswami GK. The fat pad sign. Radiology 2002;222(02):419-420

24 McCall D, Safran MR. Injuries about the shoulder in skiing and snowboarding. Br J Sports Med 2009;43(13):987-992

25 Ogawa H, Sumi H, Sumi Y, Shimizu K. Glenohumeral dislocations in snowboarding and skiing. Injury 2011;42(11):1241-1247

26 Neep MJ, Aziz A. Radiography of the acutely injured shoulder. Radiography 2011;17(03):188-192

27 Pogorzelski J, Beitzel K, Ranuccio F, et al. The acutely injured acromioclavicular joint-which imaging modalities should be used for accurate diagnosis? A systematic review. BMC Musculoskelet Disord 2017;18(01):515

28 Amini B, Beckmann NM, Beaman FD, et al; Expert Panel on Musculoskeletal Imaging. ACR Appropriateness Criteria ${ }^{\circledR}$ Shoulder Pain-Traumatic. J Am Coll Radiol 2018;15(5S)S171-S188

29 Pino EC, Colville MR. Snowboard injuries. Am J Sports Med 1989; 17(06):778-781

30 Hagel B. Skiing and snowboarding injuries. In: Caine DJ, Maffulli N, eds. Epidemiology of Pediatric Sports Injuries. Vol 48.Basel, Switzerland: Karger Publishers; 2005:74-119

31 Coury T, Napoli AM, Wilson M, Daniels J, Murray R, Milzman D. Injury patterns in recreational alpine skiing and snowboarding at a mountainside clinic. Wilderness Environ Med 2013;24(04): 417-421

32 Bladin C, Giddings P, Robinson M. Australian snowboard injury data base study. A four-year prospective study. Am J Sports Med 1993;21(05):701-704

33 Ishimaru D, Ogawa H, Sumi H, Sumi Y, Shimizu K. Lower extremity injuries in snowboarding. J Trauma 2011;70(03):E48-E52

34 Yvars MF, Kanner HR. Ski fractures of the femur. Am J Sports Med 1984;12(05):386-390

35 Bachmann LM, Kolb E, Koller MT, Steurer J, ter Riet G. Accuracy of Ottawa ankle rules to exclude fractures of the ankle and mid-foot: systematic review. BMJ 2003;326(7386):417 
36 Handel DA, Gaines SA. Ankle injuries. In: Tintinalli JE, Stapczynski JS, Ma OJ, Yealy DM, Meckler GD, Cline DM, eds. Tintinalli's Emergency Medicine: A Comprehensive Study Guide. 8th ed. New York, NY: McGraw-Hill Education; 2015:Chapter 273

37 Lin C-F, Gross ML, Weinhold P. Ankle syndesmosis injuries: anatomy, biomechanics, mechanism of injury, and clinical guidelines for diagnosis and intervention. J Orthop Sports Phys Ther 2006;36(06):372-384

38 Ryan LP, Hills MC, Chang J, Wilson CD. The lambda sign: a new radiographic indicator of latent syndesmosis instability. Foot Ankle Int 2014;35(09):903-908

39 Clark TW, Janzen DL, Ho K, Grunfeld A, Connell DG. Detection of radiographically occult ankle fractures following acute trauma: positive predictive value of an ankle effusion. AJR Am J Roentgenol 1995;164(05):1185-1189

40 Smith SE, Chang EY, Ha AS, et al; Expert Panel on Musculoskeletal Imaging. ACR Appropriateness Criteria ${ }^{\circledR}$ Acute Trauma to the Ankle. J Am Coll Radiol 2020;17(11S):S355-S366

41 Oae K, Takao M, Naito K, et al. Injury of the tibiofibular syndesmosis: value of MR imaging for diagnosis. Radiology 2003;227 (01):155-161

42 Vogl TJ, Hochmuth K, Diebold T, et al. Magnetic resonance imaging in the diagnosis of acute injured distal tibiofibular syndesmosis. Invest Radiol 1997;32(07):401-409

43 Polzer H, Kanz KG, Prall WC, et al. Diagnosis and treatment of acute ankle injuries: development of an evidence-based algorithm. Orthop Rev (Pavia) 2012;4(01):e5

44 Oae K, Takao M, Uchio Y, Ochi M. Evaluation of anterior talofibular ligament injury with stress radiography, ultrasonography and MR imaging. Skeletal Radiol 2010;39(01):41-47

45 Kirkpatrick DP, Hunter RE, Janes PC, Mastrangelo J, Nicholas RA. The snowboarder's foot and ankle. Am J Sports Med 1998;26(02): 271-277

46 Jibri Z, Mukherjee K, Kamath S, Mansour R. Frequently missed findings in acute ankle injury. Semin Musculoskelet Radiol 2013; 17(04):416-428

47 Funk JR, Srinivasan SCM, Crandall JR. Snowboarder's talus fractures experimentally produced by eversion and dorsiflexion. Am J Sports Med 2003;31(06):921-928

48 Bladin C, McCrory P. Snowboarding injuries. An overview. Sports Med 1995;19(05):358-364

49 Brown TD, Varney TE, Micheli LJ. Malleolar bursitis in figure skaters. Indications for operative and nonoperative treatment. Am J Sports Med 2000;28(01):109-111

50 Anderson SE, Weber M, Steinbach LS, Ballmer FT. Shoe rim and shoe buckle pseudotumor of the ankle in elite and professional figure skaters and snowboarders: MR imaging findings. Skeletal Radiol 2004;33(06):325-329

51 Boutin RD, Fritz RC. MRI of snow skiing and snowboarding injuries. Semin Musculoskelet Radiol 2005;9(04):360-378

52 Brown RR, Sadka Rosenberg Z, Schweitzer ME, Sheskier S, Astion D, Minkoff J. MRI of medial malleolar bursa. AJR Am J Roentgenol 2005;184(03):979-983

53 Laporte J-D, Binet M-H, Constans D. Evolution of ACL ruptures in French ski resorts 1992-1999. In: Johnson R, Zucco P, Shealy J, eds. Skiing Trauma and Safety: Thirteenth Volume. Conshohocken, PA: ASTM International; 2000

54 Médecins de Montagne. Summary of epidemiology of winter sports injuries 2007. Accessed April 20, 2008 at: http://www. mdem.org

55 Torjussen J, Bahr R. Injuries among elite snowboarders (FIS Snowboard World Cup). Br J Sports Med 2006;40(03):230-234

56 Davies H, Tietjens B, Van Sterkenburg M, Mehgan A. Anterior cruciate ligament injuries in snowboarders: a quadriceps-induced injury. Knee Surg Sports Traumatol Arthrosc 2009;17 (09):1048-1051

57 Bere T, Flørenes TW, Krosshaug T, et al. Mechanisms of anterior cruciate ligament injury in World Cup alpine skiing: a systematic video analysis of 20 cases. Am J Sports Med 2011;39(07): 1421-1429

58 O'Shea KJ, Murphy KP, Heekin RD, Herzwurm PJ. The diagnostic accuracy of history, physical examination, and radiographs in the evaluation of traumatic knee disorders. Am J Sports Med 1996;24 (02):164-167

59 Tai AW, Alparslan HL, Townsend BA, et al. Accuracy of cross-table lateral knee radiography for evaluation of joint effusions. AJR Am J Roentgenol 2009;193(04):W339-44

60 Sims JI, Chau MT, Davies JR. Diagnostic accuracy of the Ottawa Knee Rule in adult acute knee injuries: a systematic review and meta-analysis. Eur Radiol 2020;30(08):4438-4446

61 Tuite MJ, Daffner RH, Weissman BN, et al. American College of Radiology ACR Appropriateness Criteria acute trauma to the knee. J Am Coll Radiol 2012;9(02):96-103

62 Mustonen AOT, Koskinen SK, Kiuru MJ. Acute knee trauma: analysis of multidetector computed tomography findings and comparison with conventional radiography. Acta Radiol 2005; 46(08):866-874

63 Behairy NH, Dorgham MA, Khaled SA. Accuracy of routine magnetic resonance imaging in meniscal and ligamentous injuries of the knee: comparison with arthroscopy. Int Orthop 2009;33(04):961-967

64 Mirowitz SA, Shu HH. MR imaging evaluation of knee collateral ligaments and related injuries: comparison of T1-weighted, T2weighted, and fat-saturated T2-weighted sequences-correlation with clinical findings. J Magn Reson Imaging 1994;4(05):725-732

65 Van Dyck P, Vanhoenacker FM, Lambrecht V, et al. Prospective comparison of 1.5 and 3.0-T MRI for evaluating the knee menisci and ACL. J Bone Joint Surg Am 2013;95(10):916-924

66 Ogawa H, Sumi H, Sumi Y, Shimizu K. Pelvic fractures resulting from snowboarding. Am J Sports Med 2010;38(03):538-542

67 Bydon M, De la Garza-Ramos R, Macki M, Desai A, Gokaslan AK, Bydon A. Incidence of sacral fractures and in-hospital postoperative complications in the United States: an analysis of 2002-2011 data. Spine 2014;39(18):E1103-E1109

68 Davis DD, Foris LA, Kane SM, et al. Pelvic fracture. StatPearls Accessed March 19, 2021 at: https://www.ncbi.nlm.nih.gov/books/ NBK430734/

69 Resnik CS, Stackhouse DJ, Shanmuganathan K, Young JW. Diagnosis of pelvic fractures in patients with acute pelvic trauma: efficacy of plain radiographs. AJR Am J Roentgenol 1992;158(01):109-112

70 Khurana B, Sheehan SE, Sodickson AD, Weaver MJ. Pelvic ring fractures: what the orthopedic surgeon wants to know. Radiographics 2014;34(05):1317-1333

71 Hasler RM, Baschera D, Taugwalder D, Exadaktylos AK, Raabe A. Cohort study on the association between helmet use and traumatic brain injury in snowboarders from a Swiss tertiary trauma center. World Neurosurg 2015;84(03):805-812

72 Haider AH, Saleem T, Bilaniuk JW, Barraco RDEastern Association for the Surgery of Trauma Injury ControlViolence Prevention Committee. An evidence-based review: efficacy of safety helmets in the reduction of head injuries in recreational skiers and snowboarders. J Trauma Acute Care Surg 2012;73(05):1340-1347

73 Xiang H, Kelleher K, Shields BJ, Brown KJ, Smith GA. Skiing- and snowboarding-related injuries treated in U.S. emergency departments, 2002. J Trauma 2005;58(01):112-118

74 Nakaguchi H, Tsutsumi K. Mechanisms of snowboarding-related severe head injury: shear strain induced by the opposite-edge phenomenon. J Neurosurg 2002;97(03):542-548

75 National Ski Areas Association Information from the 2012/13. NSAA National Demographic Study Survey. Accessed November 25, 2020 at: https://www.nsaa.org/

76 Davis PCExpert Panel on Neurologic Imaging. Head trauma. AJNR Am J Neuroradiol 2007;28(08):1619-1621

77 Lee H, Wintermark M, Gean AD, Ghajar J, Manley GT, Mukherjee P. Focal lesions in acute mild traumatic brain injury and neurocognitive outcome: CT versus 3T MRI. J Neurotrauma 2008;25(09): 1049-1056 
68 Imaging Review of Snowboard Injuries Bohyn et al.

78 Levy AS, Smith RH. Neurologic injuries in skiers and snowboarders. Semin Neurol 2000;20(02):233-246

79 Wakahara K, Matsumoto K, Sumi H, Sumi Y, Shimizu K. Traumatic spinal cord injuries from snowboarding. Am J Sports Med 2006; 34(10):1670-1674

80 Seino H, Kawaguchi S, Sekine M, Murakami T, Yamashita T. Traumatic paraplegia in snowboarders. Spine $2001 ; 26(11): 1294-1297$
81 Koo DW, Fish WW. Spinal cord injury and snowboarding-the British Columbia experience. J Spinal Cord Med 1999;22(04): 246-251

82 Floyd T. Alpine skiing, snowboarding, and spinal trauma. Arch Orthop Trauma Surg 2001;121(08):433-436

83 Guarnieri G, Izzo R, Muto M. The role of emergency radiology in spinal trauma. Br J Radiol 2016;89(1061):20150833 